\section{Cleaning of Laundry and Trash Chutes}

To the editor:

Our hospital recently completed an extensive reconstruction program, in which laundry and trash chutes were installed.

Our Infection Control Committee would very much appreciate any information you can offer regarding the cleaning of the interior of the aforementioned chutes.

Sincerely,

Jacqueline M. Winstead, R.N. Infection Surveillance Coord. St. John's Episcopal Hospital Brooklyn, New Yorh

Dear Ms. Winstead:

There are several possible ways to do such cleaning. The most effective (and probably the most dangerous) method is to lower a very small person on a "bosun's chair" from top to bottom through the chute(s) to provide the direct physical cleaning with a properly diluted disinfectant-detergent (I'd suggest application using a "pistolgrip" sprayer) and clean rags. This method is not generally considered to be practical.

So, I've recommended what is probably a much more practical system: using a wand with a stationary or rotating brush on its end and incorporating in the wand a detergent cartridge (which can be turned on and off). The hose should be screwed onto a hose bibb at a clinical sink or hopper closest to the chute(s) on each floor. Starting with the top floor, a person cleaning the chute leans into it and washes it down from that floor to the next floor, brushing all surfaces with the stream of warm water and detergent. After doing this for sufficient time to soap down the walls, then warm tap water should be used to wash off the detergent. Then; the person should move down, floor-by-floor, repeating the same procedure. Finally, a large bag of tightly packed, cleanlinen scraps can be pulled down with a rope from the top to the bottom to remove all detergent residues or pieces of soil that have not been removed with the high-pressure washing described above. Obviously, a chute must be locked out of use when a cleaning procedure is underway, to prevent injuries to the cleaning person.

If your institution has the misfortune of having a high-velocity-vacuum-chute system, that system can be cleaned by passing tightly fitted, disinfectant-soaked bags through it several times. No matter what the type of chute, if all linens are bagged there will seldom be a need for cleaning of chutes.

I hope that these comments answer your questions. If not, or if we can attempt to be of further service at any time, please let us know.

Sincerely yours, George F. Mallison Bacterial Diseases Division Center for Infectious Diseases
After reading this letter, my initial response was to explain how to clean the laundry and trash chutes. However, I believe the problem is somewhat more complex.

First of all, I question why chutes, especially trash chutes, were even proposed. To date, there is no authentic recommendation as to how to clean these monstrosities. Most people agree that cleaning is simply impossible. This should have been an important consideration of the planning committee, which should have an infection control practitioner as a representative. "An ounce of prevention," certainly pertains to this type of situation.

No matter, the situation does exist and must be dealt with. Again, preventive measures are the answer. The following points may be helpful in developing guidelines for the maintenance of these chutes.

Laundry chutes:

1. All laundry should be bagged and closed securely before being placed in the laundry chute. The hamper must not be completely filled in order for it to close securely.

2. Laundry that is soiled with patients' excretions or secretions should be folded to confine and contain. If the laundry is soiled to the extent that confinement and containment is not possible, the soiled laundry should be initially placed into a water soluble bag, closed securely and then placed into a cloth laundry hamper and closed securely. (All plastic laundry bags must be con- 